\title{
Structural optimization of multibody system components described using level set techniques
}

\author{
Emmanuel Tromme • Daniel Tortorelli • Olivier Brüls • Pierre \\ Duysinx
}

Received: date / Accepted: date

\begin{abstract}
The structural optimization of the components in multibody systems is performed using a fully coupled optimization method. The design's predicted response is obtained from a flexible multibody system simulation under various service conditions. In this way, the resulting optimization process enhances most existing studies which are limited to weakly coupled (quasi-) static or frequency domain loading conditions. A level set description of the component geometry is used to formulate a generalized shape optimization problem which is solved via efficient gradient-based optimization methods. Gradients of cost and constraint functions are obtained from a sensitivity analysis which is revisited in order to facilitate its implementation and retain its computational efficiency. The optimizations of a slidercrank mechanism and a 2-dof robot are provided to exemplify the procedure.
\end{abstract}

Keywords Dynamic Response Optimization · Flexible Multibody Systems · Sensitivity Analysis · Level Set Description - Generalized Shape Optimization

\section{Introduction}

The optimal design of a mechanical system component is usually achieved using a component-based approach in which the component is isolated from its system and

E. Tromme · O. Brüls · P. Duysinx

University of Liege (ULg):

Aerospace and Mechanical Engineering Department

E-mail: $\{$ emmanuel.tromme;o.bruls;p.duysinx $\} @ u l g . a c . b e$

D. Tortorelli

University of Illinois at Urbana-Champaign

Department of Mechanical Science and Engineering

E-mail: dtortore@illinois.edu subjected to boundary and loading conditions to predict stress distributions etc. These loading conditions generally come from the designer experience, empirical relations or experimental efforts. And even though the majority of loads are dynamic, structural optimization typically treats such loadings as (quasi-)static or frequency domain loadings due to the difficulties of evaluating the dynamic response and then incorporating it in the optimization.

The component-based approach has been extended to a system-level approach which incorporates a multibody system (MBS) simulation to evaluate the response of the whole system (Bruns and Tortorelli 1995; Oral and Kemal Ider 1997). This extension is important because the optimal component designs may be very sensitive to the support and loading conditions (Bendsøe and Sigmund 2003).

To perform the dynamic response optimization of a component, the weakly coupled method reformulates the problem using a two step approach. Firstly, initially a rigid but later flexible MBS simulation computes the loads applied to each component and secondly, each component is optimized independently using a quasistatic approach in which a series of equivalent static load cases obtained from the MBS simulation are applied to the respective components (Kang et al 2005). Several works used this method (Häussler et al 2004; Kang et al 2005; Hong et al 2010; Sherif and Irschik 2010). Häussler et al (2001) showed that it is important to consider the load inertia property relationship during the optimization process since these interactions might be significant.

In the fully coupled approach, Brüls et al (2011) took advantage of the evolution of numerical simulations and topology optimization to design structural components within a flexible MBS simulation. They 
showed the feasibility and convenience of integrating the optimization loop directly to the flexible MBS simulation. In this way, the dynamic effects are naturally incorporated into the design.

Tromme et al (2013) further investigated the fully coupled approach and showed that the formulation is essential to obtain good convergence of the optimization. Indeed, significant coupling between vibration and component inertial properties plus the interactions between flexible components renders a complex design problem which suffers poor convergence if not treated properly.

Seifried and Held (2011) use the fully coupled approach to optimize controlled flexible multibody systems. Durability-based constraints are considered in the fully coupled work of Tobias et al (2010). They avoid post-processing the elastic multibody system simulation results by evaluating the damage values during the elastic multibody system simulation to hasten their computations.

In contrast to the weakly coupled method, the fully coupled method uses the time response obtained from the flexible MBS analysis to define optimization cost and constraint functions. Hence, the behavior of the entire mechanical system is considered in the optimization problem, i.e. it is not limited to the behavior of the optimized component. Moreover, this approach offers a global-local view of the problem which is necessary when designing lighter - more flexible components. Indeed, reducing the mass and thus increasing the flexibility of a single component can drastically influence the overall mechanical system behavior. The fully coupled approach evaluates the dynamic loading exerted on the considered component (local approach) whereas the optimization considers system behavior (global approach).

In this paper, the components in a multibody system system are optimized using the fully coupled approach. The implicit representation of the component geometry is achieved using the level set description (Osher and Sethian 1988). This representation enables a generalized shape optimization wherein the component topology can be changed on a limited basis. Finally, a novel semi-analytical algorithm is presented to facilitate the efficient computation of the design sensitivities.

A major problem in shape optimization is mesh distortion which occurs during the optimization. The regular mesh of the initial design is distorted after a few design iterations whereupon tangled and sliver elements appear. Thusly, the accuracy of the simulated response decreases. Adaptative mesh algorithms have been developed to combat this problem, however the re-meshing operations are time consuming (Haftka and Grandhi
1986; Duysinx et al 1994; Schleupen et al 2000). Moreover adaptative mesh techniques produce discontinuities in the objective function and/or constraints between optimization iterations which is undesirable in gradient-based algorithms (Van Keulen et al 2005).

Topology optimization has been developed to determine the optimal design of a component without a priori information of the component layout (Bendsøe and Kikuchi 1988). It only requires the definitions of the spatial design domain, material properties, boundary conditions and load cases, making this method more powerful than sizing or shape optimization. Topology optimization also works on a fixed mesh. Thusly it avoids the previously mentioned mesh distortion issues. However, since a volume fraction design variable is associated with each finite element in the mesh, the number of variables is large making it a complex optimization problem with many local optima. Moreover, the optimal design boundary is fuzzy making the final design open to subjective interpretation.

The present approach combines the advantages of shape and topology optimizations by 1) working on a fixed mesh to avoid the mesh distortion, 2) having a precise description of the component geometry with a reasonable number of parameters and 3) providing the ability to change topology in a limited manner.

The first two parts of the paper discuss the level set description and the flexible multibody system analysis, i.e. the derivation of the equations of motion and the time integration scheme. Afterwards, the optimization process is detailed in three parts: the optimization problem formulation, the optimization algorithm and the design sensitivity analysis. Special care is given to the sensitivity analysis which requires the solution of a pseudo problem using the linearized equations of motion. Unfortunately, the matrices of these linearized equations are not often available and the pseudo-load computation is painstaking to evaluate. In this paper, a novel method based on the semi-analytical method is implemented to efficiently and simplistically perform the sensitivity analysis. Finally, the optimizations of a connecting rod in a reciprocating engine with cyclic dynamic loading and a 2-dof robot subject to a trajectory tracking constraint illustrate the approach's feasibility and show its potential to optimize both the component shape and topology.

\section{Level Set Description of the Geometry}

\subsection{Generality}

In shape optimization the domain boundary is traditionally described explicitly, e.g. using splines. More 
recently, the level set method has been used to implicitly represent the boundary (Osher and Sethian 1988). Here we combine the methods. To do this, we introduce a smooth scalar-valued function $\phi: D \in \mathbb{R}^{n} \times \mathbb{R}^{n_{v}} \rightarrow \mathbb{R}$, the level set function (LSF), to represent the boundary via the dimension $n-1$ set $\{\mathbf{x} \in D: \phi(\mathbf{x}, \mathbf{p})=0\}$ where $D \in \mathbb{R}^{n}$ is the design domain and $\mathbf{p}$ is an element of the parameter space $\mathbb{R}^{n_{v}}$, that defines the spatial domain $\Omega$. The latter domain, the void domain $D \backslash \Omega$ and the domain boundary $\partial \Omega$ are defined as

$$
\left\{\begin{array}{l}
\phi(\mathbf{x}, \mathbf{p})>0, \Leftrightarrow \mathbf{x} \in \Omega \text { (spatial) } \\
\phi(\mathbf{x}, \mathbf{p})<0, \Leftrightarrow \mathbf{x} \in D \backslash \Omega \text { (void) } \\
\phi(\mathbf{x}, \mathbf{p})=0, \Leftrightarrow \mathbf{x} \in \partial \Omega \text { (interface). }
\end{array}\right.
$$

Modifying the LSF parameter $\mathbf{p}$ alters both shape and topology of the domain $\Omega$.

The next two sections describe the parameterization of the LSF and the geometry mapping adopted. They are based on the level set method review article (Van Dijk et al 2013).

\subsection{Parameterization}

The level set function can be written in its most general form as

$\phi=\phi(\mathbf{x}, \psi(\mathbf{x}, \mathbf{p}))$

where $\psi$ is an auxiliary field that may be used to introduce a minimum length scale for instance. In this paper, the LSF is parameterized using a combination of geometric features as introduced by Van Miegroet and Duysinx (2007). Boolean operations are applied to the individual features to obtain the final component geometry, i.e. we use Constructive Solid Geometry (Shapiro 2007). Each feature has its own LSF and the LSF $\phi$ of (1) is defined via the signed-distance function from all of the features. In the case of simple geometric features, these distance functions are analytical. Figures 1(a)1 (c) illustrate the creation of a cross hole in a square plate by combining two super-ellipse features.

This LSF parameterization has several advantages. First, the number of design variables $n_{v}$ is relatively small as it equals the number of geometric parameters that define the individual features. Second, since the geometric features are based on CAD entities (circle, ellipse, NURBS...), the optimal design can be manufactured without any subjective post-processing step. Third, minimum feature sizes can be accommodated by limiting the number of features and constraining their sizes.
We note that this is not a true topology optimization since the number of holes in the geometry is introduced a priori. As such, topological changes are limited to holes merging. Because holes cannot be nucleated, the best topology may be absent from the design space. Future work using the topological derivative can remedy this limitation.

\subsection{Geometry representation}

In this work, the mechanical components are modeled using the finite element method. To do this, the LSF geometry must be mapped to the finite element mesh.

The LSF mapping uses a volume fraction based Eulerian approach (Norato et al 2004). The finite element mesh is fixed throughout the optimization iterations wherein the design domain "flows" through the mesh. A volume fraction variable $\mu_{e}$ is associated with each finite element. As in topology optimization, the volume fraction variables determine the element material properties. Specifically, designing with a material with density $\rho^{0}$ and Young modulus $E^{0}$, we assign the element $e$ material properties via simple interpolation as

$$
\begin{gathered}
\rho_{e}=\mu_{e} \rho^{0} \\
E_{e}=\mu_{e} E^{0},
\end{gathered}
$$

for $e=1 \ldots N_{e}$ where $N_{e}$ is the number of elements. The values of the volume fraction $\mu_{e}$ are in the interval $\left[\mu_{m i n}, 1\right]$ where $\mu_{\text {min }}$ is a small value (e.g. 0.001) strictly greater than 0 to avoid ill-conditioned stiffness matrices. Note that no SIMP or RAMP penalization is necessary to achieve black-and-white designs (Norato et al 2004).

The element volume fractions are defined by interpolating the element node LSF values. When all the element node values are positive, the element is interior to the material domain $\Omega$ and solid material properties are assigned, i.e. $\mu_{e}=1$. In the opposite case, where only negative nodal values are observed, void material properties are assigned, i.e. $\mu_{e}=\mu_{\min }$.

In mixed elements in which positive and negative nodal values appear, the design domain boundary intersects the element. In such cases, an intermediate material is assigned whose the properties are interpolated by the volume fraction $\mu_{e}$ which is defined as

$\mu_{e}=\frac{1}{V_{e}} \int_{V e} H^{*}(\phi(\mathbf{x})) d V_{e}$,

where $H^{*}$ is a smooth approximation of the Heaviside step function and $\phi(\mathbf{x})$ is interpolated by the element shape functions and level set nodal values in the usual 


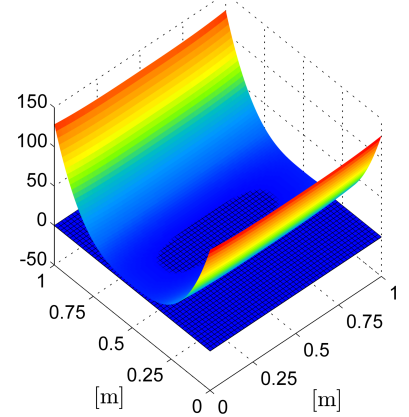

(a) Implicit representation of a first super-ellipse feature.

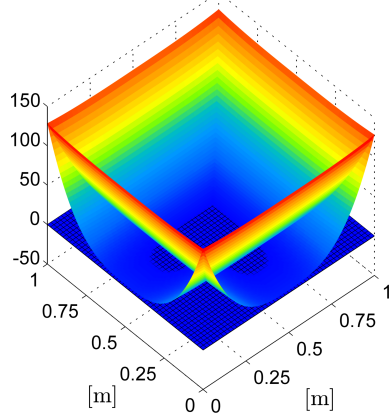

(b) Implicit representation of a second super-ellipse feature.

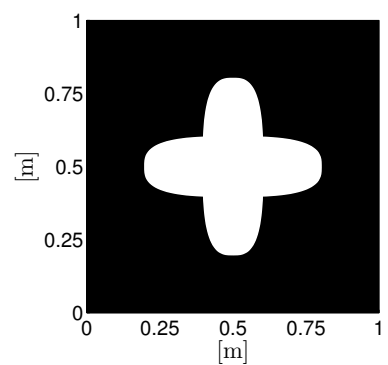

(c) Exact geometry of the cross hole.

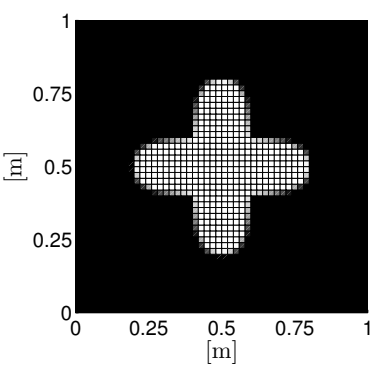

(d) Mapping resulting of the boolean operation $A N D$ of the two LSF's.

Fig. 1 Geometrical description of a cross hole in a square plate.

manner. For instance, the Heaviside step function can be approximated by the logistic function, i.e.

$H^{*}(y)=\frac{C}{1+e^{-c\left(y-y_{0}\right)}}$

where $e$ is the natural logarithm base and $y_{0}, c$, and $C$ are constants.

Error enters our finite element model since the boundary is smeared over the mixed elements due to the interpolation of $\phi$ and the approximated Heaviside step function. To reduce this error, a refined mesh is introduced inside each mixed finite element. The value of the LSF is computed for each node of this refined grid using the signed distance function. The volume fraction of the original mixed element is obtained by standard finite element interpolation over the refined grid.

In Fig. 1(d), the mapping of a cross hole in a square plate is illustrated. In static structural optimization, Kim and Chang (2005) used a similar mapping to realize "Eulerian shape optimization" wherein the geometry was defined explicitly.

\section{Flexible Multibody System Dynamics}

\subsection{Equations of motion}

In this paper, flexible multibody systems are modeled using a nonlinear finite element formulation as suggested by Géradin and Cardona (2001) which is based on an inertial frame, i.e. we use a Lagrangian approach.

Absolute nodal coordinates which correspond to the nodal displacements and orientations are gathered in the generalized coordinate vector $\mathbf{q}$. If the multibody system is not constrained, its motion is obtained by solving the equation

$\mathbf{M}(\mathbf{q}) \ddot{\mathbf{q}}=\mathbf{g}(\dot{\mathbf{q}}, \mathbf{q}, t)=\mathbf{g}^{e x t}-\mathbf{g}^{i n t}-\mathbf{g}^{g y r}$ subject to the initial conditions where $\mathbf{M}$ is the mass matrix, $\ddot{\mathbf{q}}, \dot{\mathbf{q}}$ and $\mathbf{q}$ are respectively the generalized acceleration, velocity and displacement vectors, and $\mathbf{g}^{e x t}, \mathbf{g}^{i n t}$ and $\mathbf{g}^{g y r}$ are the generalized external, internal and complementary inertia forces. It is noted that the mass matrix can depend on the generalized coordinates.

In our MBS simulation, kinematic constraints, denoted by $\mathbf{\Phi}(\mathbf{q}, t)=\mathbf{0}$, are enforced to ensure the connections between bodies due to hinges, spherical joints, etc. For simplicity only holonomic constraints are considered in this work.

The addition of the kinematic constraints to (6) yield a system of differential algebraic equations (DAE). Our formulation uses the augmented Lagrangian approach to incorporate the constraints whereupon we solve

$$
\begin{aligned}
\mathbf{M}(\mathbf{q}) \ddot{\mathbf{q}}+\mathbf{\Phi}_{q}^{T}(\mathbf{q}, t)(k \boldsymbol{\lambda}+\zeta \mathbf{\Phi}(\mathbf{q}, t)) & =\mathbf{g}(\dot{\mathbf{q}}, \mathbf{q}, t) \\
k \boldsymbol{\Phi}(\mathbf{q}, t) & =\mathbf{0},
\end{aligned}
$$

where $\zeta$ is a penalty factor, $\boldsymbol{\lambda}$ is the Lagrangian multiplier vector, $k$ is a scaling factor and $\boldsymbol{\Phi}_{q}$ is the gradient of the constraint vector. The penalty term $\zeta \boldsymbol{\Phi}_{q}^{T} \boldsymbol{\Phi}$ added in (7) facilitates the convergence of the solver. However, since this term vanishes at convergence, the response of the system is independent of the choice of $\zeta$.

\subsection{Time integration}

To solve the set of nonlinear DAE (7), Géradin and Cardona (2001) use the generalized- $\alpha$ time integration scheme initially developed by Chung and Hulbert (1993). Arnold and Brüls (2007) demonstrate that, despite the presence of algebraic constraints and the non-constant character of the mass matrix, this scheme leads to accurate and reliable results provided a small amount of numerical damping is added.

$\mathbf{q}(0)=\mathbf{q}_{0}$ and $\dot{\mathbf{q}}(0)=\dot{\mathbf{q}}_{0}$ 
According to the generalized- $\alpha$ method, a vector a of acceleration-like variables is defined by the following recursion relation

$$
\left(1-\alpha_{m}\right) \mathbf{a}_{n+1}+\alpha_{m} \mathbf{a}_{n}=\left(1-\alpha_{f}\right) \ddot{\mathbf{q}}_{n+1}+\alpha_{f} \ddot{\mathbf{q}}_{n},
$$

with $\mathbf{a}_{0}=\ddot{\mathbf{q}}_{0}$. The time integration scheme is subsequently obtained by employing $\mathbf{a}$ in the Newmark integration formulae:

$\mathbf{q}_{n+1}=\mathbf{q}_{n}+h \dot{\mathbf{q}}_{n}+h^{2}\left(\frac{1}{2}-\beta\right) \mathbf{a}_{n}+h^{2} \beta \mathbf{a}_{n+1}$

$\dot{\mathbf{q}}_{n+1}=\dot{\mathbf{q}}_{n}+h(1-\gamma) \mathbf{a}_{n}+h \gamma \mathbf{a}_{n+1}$

where $h$ denotes the time step. If the parameters $\alpha_{f}$, $\alpha_{m}, \beta$ and $\gamma$ are properly chosen according to Chung and Hulbert (1993), second-order accuracy and unconditional stability are guaranteed for linear problems. It is convenient to define these parameters in terms of the spectral radius at infinite frequencies $\rho_{\infty} \in[0,1]$ as

$$
\begin{gathered}
\alpha_{m}=\frac{2 \rho_{\infty}-1}{\rho_{\infty}+1}, \quad \alpha_{f}=\frac{\rho_{\infty}}{\rho_{\infty}+1}, \\
\gamma=\frac{1}{2}-\alpha_{m}+\alpha_{f}, \quad \beta=\frac{1}{4}\left(\gamma+\frac{1}{2}\right)^{2} .
\end{gathered}
$$

The choice $\rho_{\infty}=0$ annihilates high frequency whereas $\rho_{\infty}=1$ corresponds to no numerical damping.

A Newton-Raphson procedure is employed to solve the implicit system of equations (7) at time $t_{n+1}$. Using a predictor-corrector scheme, the unknown response $(\ddot{\mathbf{q}}, \dot{\mathbf{q}}, \mathbf{q}, \boldsymbol{\lambda})$ is divided into an approximate solution $\left(\mathbf{q}^{*}, \dot{\mathbf{q}}^{*}, \ddot{\mathbf{q}}^{*}, \boldsymbol{\lambda}^{*}\right)$ and a correction $(\Delta \mathbf{q}, \Delta \dot{\mathbf{q}}, \Delta \ddot{\mathbf{q}}, \Delta \boldsymbol{\lambda})$ leading to

$\mathbf{q} \rightarrow \mathbf{q}^{*}+\Delta \mathbf{q}$,

$\dot{\mathbf{q}} \rightarrow \dot{\mathbf{q}}^{*}+\Delta \dot{\mathbf{q}}$,

$\ddot{\mathrm{q}} \rightarrow \ddot{\mathrm{q}}^{*}+\Delta \ddot{\mathrm{q}}$,

$\lambda \rightarrow \lambda^{*}+\Delta \lambda$.

where we drop the $n+1$ subscript. The correction is computed based on the linearized form of the residual equation around the approximate solution

$$
\begin{gathered}
{\left[\begin{array}{c}
\mathbf{r}^{l i n}\left(\mathbf{q}^{*}+\Delta \mathbf{q}, \dot{\mathbf{q}}^{*}+\Delta \dot{\mathbf{q}}, \ddot{\mathbf{q}}^{*}+\Delta \ddot{\mathbf{q}}, \boldsymbol{\lambda}^{*}+\Delta \boldsymbol{\lambda}, t\right) \\
k \boldsymbol{\Phi}^{l i n}\left(\mathbf{q}^{*}+\Delta \mathbf{q}, t\right)
\end{array}\right] \approx} \\
{\left[\begin{array}{c}
\mathbf{r}^{*}\left(\mathbf{q}^{*}, \dot{\mathbf{q}}^{*}, \ddot{\mathbf{q}}^{*}, \boldsymbol{\lambda}^{*}, t\right) \\
k \boldsymbol{\Phi}^{*}\left(\mathbf{q}^{*}, t\right)
\end{array}\right]+\left[\begin{array}{cc}
\mathbf{M} & \mathbf{0} \\
\mathbf{0} & \mathbf{0}
\end{array}\right]\left[\begin{array}{c}
\Delta \ddot{\mathbf{q}} \\
\Delta \boldsymbol{\lambda}
\end{array}\right]+} \\
{\left[\begin{array}{cc}
\mathbf{C}_{t} & \mathbf{0} \\
\mathbf{0} & \mathbf{0}
\end{array}\right]\left[\begin{array}{c}
\Delta \dot{\mathbf{q}} \\
\Delta \boldsymbol{\lambda}
\end{array}\right]+\left[\begin{array}{cc}
\mathbf{K}_{t} & k \boldsymbol{\Phi}_{q}^{T} \\
k \boldsymbol{\Phi}_{q} & \mathbf{0}
\end{array}\right]\left[\begin{array}{c}
\Delta \mathbf{q} \\
\Delta \boldsymbol{\lambda}
\end{array}\right]=\mathbf{0},}
\end{gathered}
$$

where $\mathbf{C}_{t}=\partial \mathbf{r} / \partial \dot{\mathbf{q}}$ and $\mathbf{K}_{t}=\partial \mathbf{r} / \partial \mathbf{q}$ denote respectively the tangent damping and tangent stiffness matrices and $\mathbf{r}=\mathbf{M} \ddot{\mathbf{q}}-\mathbf{g}+\boldsymbol{\Phi}_{\mathbf{q}}^{T}(k \boldsymbol{\lambda}+\zeta \boldsymbol{\Phi})$ is the residual corresponding to $(7)$.
To solve (16), the integration formulae (8)-(10) are manipulated by solving (8) for $\mathbf{a}_{n+1}$ and inserting that result into (9) and (10). The resulting two equations are

$$
\begin{aligned}
\Delta \dot{\mathbf{q}} & =\frac{\gamma}{\beta h} \Delta \mathbf{q} \\
\Delta \ddot{\mathbf{q}} & =\frac{1}{\beta h^{2}} \frac{1-\alpha_{m}}{1-\alpha_{f}} \Delta \mathbf{q} .
\end{aligned}
$$

Using (17)-(18), the linearized form of the update equation (16) reduces to

$$
\mathbf{S}_{t}\left[\begin{array}{c}
\Delta \mathbf{q} \\
\Delta \boldsymbol{\lambda}
\end{array}\right]=-\left[\begin{array}{c}
\mathbf{r}^{*} \\
k \mathbf{\Phi}^{*}
\end{array}\right]
$$

where $\mathbf{S}_{t}$ is the tangent iteration matrix defined as

$\mathbf{S}_{t}=\left[\begin{array}{cc}\frac{1-\alpha_{m}}{1-\alpha_{f}} \frac{1}{\beta h^{2}} \mathbf{M}+\frac{\gamma}{\beta h} \mathbf{C}_{t}+\mathbf{K}_{t} & k \boldsymbol{\Phi}_{\mathbf{q}}^{T} \\ k \mathbf{\Phi}_{\mathbf{q}} & \mathbf{0}\end{array}\right]$

Equation (19) is solved for the correction $(\Delta \mathbf{q}, \Delta \boldsymbol{\lambda})$ and the approximate solution is updated, cf. (12)-(15) and (17)-(18). Iterations continue until the residuals of (7), $\mathbf{r}$ and $\boldsymbol{\Phi}$, are close to zero, i.e. until $|\mathbf{r}|<\operatorname{tol}_{\mathbf{r}}$ and $|\boldsymbol{\Phi}|<$ tol $_{\boldsymbol{\Phi}}$.

Algorithm 1 summarizes the previous developments. We note that the process is initialized with $\boldsymbol{\lambda}_{n+1}=\mathbf{0}$ and $\ddot{\mathbf{q}}_{n+1}=\mathbf{0}$. Initialization with the previous time step, i.e. $\ddot{\mathbf{q}}_{n+1}=\ddot{\mathbf{q}}_{n}$ and $\boldsymbol{\lambda}_{n+1}=\boldsymbol{\lambda}_{n}$, is also possible but does not significantly improve the convergence speed. The condition $i t<i t_{\max }$ limits the number of iterations inside the iterative process.

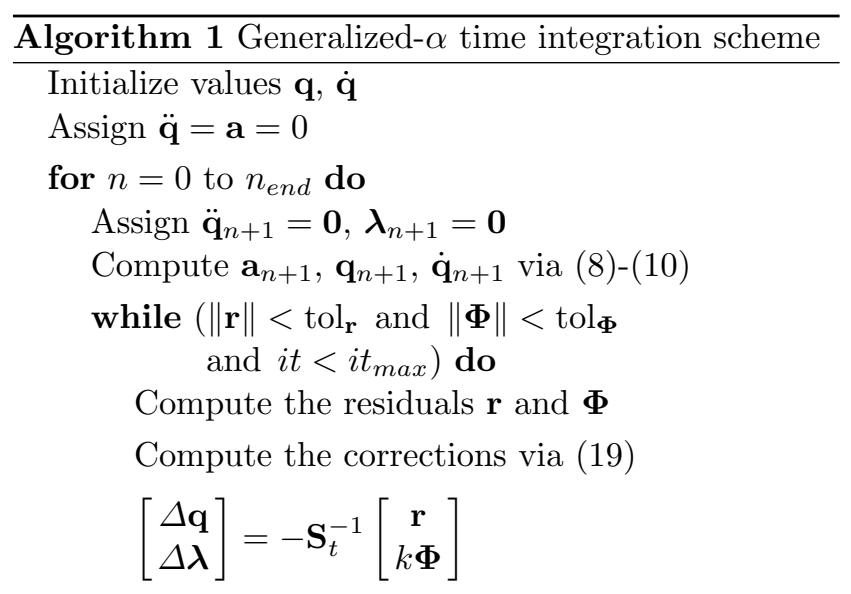

Increment $\mathbf{q}, \dot{\mathbf{q}}, \ddot{\mathbf{q}}, \boldsymbol{\lambda}$ via (12)-(15) and (17)-(18) end while $\mathbf{a}_{n+1}=\mathbf{a}_{n+1}+\frac{1-\alpha_{f}}{1-\alpha_{m}} \ddot{\mathbf{q}}_{n+1}$, cf. (8)

end for 


\section{Optimization of Flexible Multibody Systems}

\subsection{Formulation of the MBS optimization problem}

The optimization problem concerns the minimization of an objective function $f_{0}(\mathbf{s}, t)$ subjected to $m$ constraints $f_{j}(\mathbf{s}, t) \leq \bar{f}_{j}$ which ensure the integrity of the structural design and its manufacturability. The vector

$\mathbf{s}=[\mathbf{q}, \dot{\mathbf{q}}, \ddot{\mathbf{q}}, \lambda, \mathbf{p}]$

gathers the dependent variables $\mathbf{q}, \dot{\mathbf{q}}, \ddot{\mathbf{q}}$ and $\boldsymbol{\lambda}$ of the optimization process, i.e. those defining the response of the MBS and the $n_{v}$ independent design variables $p_{i}$ that are modified by the optimization. Side-constraints $\underline{p}_{i} \leq p_{i} \leq \bar{p}_{i}$ which reflect technological considerations limit the design space. Mathematically, the optimization problem reads

$$
\begin{array}{cl}
\underset{\mathbf{p}}{\operatorname{minimize}} & f_{0}(\mathbf{s}, t) \\
\text { subject to } & f_{j}(\mathbf{s}, t) \leq \bar{f}_{j}, \quad j=1, \ldots, m, \\
& \underline{p}_{i} \leq p_{i} \leq \bar{p}_{i}, \quad i=1, \ldots, n_{v} .
\end{array}
$$

This problem formulation provides a general and robust design framework that is solved by various types of optimization algorithms.

In our case, the functions $f_{j}(\mathbf{s}, t)$ concern mass and displacement or stress measures at particular time instants. The design variables $p_{i}$ are the geometric parameters that define the level set functions.

This study investigates local and global formulations of the optimization problem. Local formulations enforce constraints at each time step. This option offers a tight control over the optimized design at the expense of many constraints which hinders the optimization convergence. Global formulations agglomerate the response trajectories into a few constraints. These global constraints are quite nonlinear, nonetheless the reduced number of constraints generally facilitates the optimization convergence. Unfortunately, their global nature relinquishes the precise response control at each time step. The formulations are further explained in Section 5-6.

\subsection{Optimization algorithms}

Mathematical programming tools are used to solve the optimization problem due to their success in solving large scale structural and multidisciplinary optimization problems (Sigmund and Maute 2013; Deaton and Grandhi 2014). The major advantages of these methods are their high convergence rates which limit the number of function evaluations i.e. MBS simulations, required to obtain an optimal design. The drawbacks of gradient-based algorithms is that they require gradient computations of the cost and constraint functions. As with zero-order methods, they are also sensitive to local optima and may not converge when attempting to solve highly nonlinear problems. In this study, we use the Method of Moving Asymptotes which is based on sequential convex programming (Svanberg 1987).

\subsection{Sensitivity analysis}

Gradient-based optimization methods require a sensitivity analysis to compute the derivatives of the cost and constraint functions. The sensitivity analysis efficiency is essential in the optimization process because it can drastically affect the computation time, especially when the simulation is large, as encountered with MBS analysis.

Finite difference sensitivity analysis methods are easy to implement and can accommodate almost all types of functions. While they are suitable for our purposes, we opt for another approach due to their inefficiency. Indeed, finite difference methods require one additional simulation per design variable at each optimization iterations and therefore, the CPU grows by a factor $n_{v}+1$. This is especially pertinent here since the MBS simulation time is much larger than that of a static analysis. For this reason, a semi-analytical sensitivity analysis is implemented due to its trade-off between ease of implementation and computation efficiency.

Let us consider the generic response function

$f=f(\mathbf{q}(\mathbf{p}), \dot{\mathbf{q}}(\mathbf{p}), \ddot{\mathbf{q}}(\mathbf{p}), \boldsymbol{\lambda}(\mathbf{p}), \mathbf{p})$.

The total derivative of (23) with respect to the design variable $p_{i}$ is

$$
\frac{d f}{d p_{i}}=\frac{\partial f}{\partial \mathbf{q}} \frac{d \mathbf{q}}{d p_{i}}+\frac{\partial f}{\partial \dot{\mathbf{q}}} \frac{d \dot{\mathbf{q}}}{d p_{i}}+\frac{\partial f}{\partial \ddot{\mathbf{q}}} \frac{d \ddot{\mathbf{q}}}{d p_{i}}+\frac{\partial f}{\partial \boldsymbol{\lambda}} \frac{d \boldsymbol{\lambda}}{d p_{i}}+\frac{d f}{d p_{i}}
$$

where the response derivatives are evaluated in the dynamic response sensitivity analysis detailed below.

It is well known that if the number of design variables is less than the number of functions (e.g. in a local formulation), a direct sensitivity method is preferred, otherwise an adjoint method is favored. However, in transient simulations, terminal value adjoint simulations are cumbersome to implement. Thusly, we adopt the direct method.

When the analysis converges at time step $t_{n+1}$, the mechanical system satisfies the equation of motion (7) which is repeated here to denote its dependence on $\mathbf{p}$

$$
\begin{array}{r}
\mathbf{r}\left(\mathbf{q}(\mathbf{p}), \dot{\mathbf{q}}(\mathbf{p}), \ddot{\mathbf{q}}(\mathbf{p}), \boldsymbol{\lambda}(\mathbf{p}), t_{n+1}, \mathbf{p}\right)=\mathbf{0}, \\
k \boldsymbol{\Phi}\left(\mathbf{q}(\mathbf{p}), t_{n+1}, \mathbf{p}\right)=\mathbf{0} .
\end{array}
$$


Differentiating the above yields

$\mathbf{0}=\frac{\partial \mathbf{r}}{\partial \mathbf{q}} \frac{d \mathbf{q}}{d p_{i}}+\frac{\partial \mathbf{r}}{\partial \dot{\mathbf{q}}} \frac{d \dot{\mathbf{q}}}{d p_{i}}+\frac{\partial \mathbf{r}}{\partial \ddot{\mathbf{q}}} \frac{d \ddot{\mathbf{q}}}{d p_{i}}+\frac{\partial \mathbf{r}}{\partial \boldsymbol{\lambda}} \frac{d \boldsymbol{\lambda}}{d p_{i}}+\frac{\partial \mathbf{r}}{\partial p_{i}}$,

$\mathbf{0}=k \frac{\partial \boldsymbol{\Phi}}{\partial \mathbf{q}} \frac{d \mathbf{q}}{d p_{i}}+k \frac{\partial \boldsymbol{\Phi}}{\partial p_{i}}$.

Noting that

$\frac{\partial \mathbf{r}}{\partial \mathbf{q}}=\mathbf{K}_{t}, \quad \frac{\partial \mathbf{r}}{\partial \dot{\mathbf{q}}}=\mathbf{C}_{t}, \quad \frac{\partial \mathbf{r}}{\partial \ddot{\mathbf{q}}}=\mathbf{M}, \quad \frac{\partial \mathbf{r}}{\partial \boldsymbol{\lambda}}=k \boldsymbol{\Phi}_{\mathbf{q}}^{T}$

and rearranging Equations (26)-(27) gives

$$
\begin{aligned}
\mathbf{M} \frac{d \ddot{\mathbf{q}}}{d p_{i}}+\mathbf{C}_{t} \frac{d \dot{\mathbf{q}}}{d p_{i}}+\mathbf{K}_{t} \frac{d \mathbf{q}}{d p_{i}}+k \boldsymbol{\Phi}_{\mathbf{q}}^{T} \frac{d \boldsymbol{\lambda}}{d p_{i}} & =-\frac{\partial \mathbf{r}}{\partial p_{i}}, \\
k \boldsymbol{\Phi}_{\mathbf{q}} \frac{d \mathbf{q}}{d p_{i}} & =-k \frac{\partial \boldsymbol{\Phi}}{\partial p_{i}} .
\end{aligned}
$$

Note that the resulting system of equations is linear with respect to the response derivatives $d \mathbf{q} / d p_{i}, d \dot{\mathbf{q}} / d p_{i}$, $d \ddot{\mathbf{q}} / d p_{i}$ and $d \boldsymbol{\lambda} / d p_{i}$.

As previously mentioned, we use the semi-analytical method to resolve (28). This approach has already been investigated in the context of flexible multibody systems (Bestle and Seybold 1992; Brüls and Eberhard 2008). Indeed, Brüls and Eberhard (2008) demonstrate that the sensitivity analysis can be integrated into the generalized- $\alpha$ scheme, however all the terms of (28) have to be computed. The pseudo-loads, $\partial \mathbf{r} / \partial p_{i}$ and $\partial \Phi / \partial p_{i}$, are computed using a finite difference method which requires $n_{v}$ evaluations of the residual $\left.\mathbf{r}\right|_{\mathbf{p}+\Delta \mathbf{p}_{i}}$ and $\left.\boldsymbol{\Phi}\right|_{\mathbf{p}+\Delta \mathbf{p}_{i}}$ at each time step where $\Delta \mathbf{p}_{i}=\left[0 \ldots 0, \Delta p_{i}, 0 \ldots 0\right]$. Furthermore, the matrices $\mathbf{M}, \mathbf{C}_{t}$ and $\mathbf{K}_{t}$ must be computed even though they are not usually computed independently during the primal analysis; rather they are usually aggregated in the tangent iteration matrix $\mathbf{S}_{t}$, see (19).

An alternative sensitivity formulation is proposed to ease the computational solution of (28). In our semianalytical implementation, we rearrange (26) as

$-\frac{\partial \mathbf{r}}{\partial \mathbf{q}} \frac{d \mathbf{q}}{d p_{i}}-\frac{\partial \mathbf{r}}{\partial \boldsymbol{\lambda}} \frac{d \boldsymbol{\lambda}}{d p_{i}}=\frac{\partial \mathbf{r}}{\partial \dot{\mathbf{q}}} \frac{d \dot{\mathbf{q}}}{d p_{i}}+\frac{\partial \mathbf{r}}{\partial \ddot{\mathbf{q}}} \frac{d \ddot{\mathbf{q}}}{d p_{i}}+\frac{\partial \mathbf{r}}{\partial p_{i}}$.

Note that the right-hand-side is the derivative of the residual with respect to $p_{i}$ holding $\mathbf{q}$ and $\boldsymbol{\lambda}$ fixed. This term is approximated as

$$
\begin{aligned}
\frac{\partial \mathbf{r}}{\partial \dot{\mathbf{q}}} \frac{d \dot{\mathbf{q}}}{d p_{i}}+\frac{\partial \mathbf{r}}{\partial \ddot{\mathbf{q}}} \frac{d \ddot{\mathbf{q}}}{d p_{i}}+\frac{\partial \mathbf{r}}{\partial p_{i}} \approx \\
\quad \frac{\mathbf{r}\left(\mathbf{q}(\mathbf{p}), \dot{\mathbf{q}}\left(\mathbf{p}^{*}\right), \ddot{\mathbf{q}}\left(\mathbf{p}^{*}\right), \boldsymbol{\lambda}(\mathbf{p}), t_{n+1}, \mathbf{p}^{*}\right)-\left.\mathbf{r}\right|_{\left(t_{n+1}, \mathbf{p}\right)}}{\Delta p_{i}}
\end{aligned}
$$

for "small" $\Delta p_{i}$, where we use (25) and define $\mathbf{p}^{*}=$ $\mathbf{p}+\Delta \mathbf{p}_{i}$ with $\Delta \mathbf{p}_{i}=\left[0 \ldots 0, \Delta p_{i}, 0 \ldots 0\right]$.
Substituting a first order approximation of $\left.\dot{\mathbf{q}}\right|_{\mathbf{p}^{*}} \approx$ $\left.\dot{\mathbf{q}}\right|_{\mathbf{p}}+d \dot{\mathbf{q}} /\left.d p_{i}\right|_{\mathbf{p}} \Delta p_{i}$ and $\left.\left.\ddot{\mathbf{q}}\right|_{\mathbf{p}^{*}} \approx \ddot{\mathbf{q}}\right|_{\mathbf{p}}+d \ddot{\mathbf{q}} /\left.d p_{i}\right|_{\mathbf{p}} \Delta p_{i}$ into (30) transforms (29) to

$-\frac{\partial \mathbf{r}}{\partial \mathbf{q}} \frac{d \mathbf{q}}{d p_{i}}-\frac{\partial \mathbf{r}}{\partial \boldsymbol{\lambda}} \frac{d \boldsymbol{\lambda}}{d p_{i}} \approx \frac{1}{\Delta p_{i}} \mathbf{r}_{p e r t}$,

where

$$
\begin{aligned}
\mathbf{r}_{\text {pert }}=\mathbf{r}\left(\left.\mathbf{q}\right|_{\mathbf{p}},\left.\dot{\mathbf{q}}\right|_{\mathbf{p}}+\left.\frac{d \dot{\mathbf{q}}}{d p_{i}}\right|_{\mathbf{p}} \Delta p_{i}, \ldots\right. \\
\left.\left.\ddot{\mathbf{q}}\right|_{\mathbf{p}}+\left.\frac{d \ddot{\mathbf{q}}}{d p_{i}}\right|_{\mathbf{p}} \Delta p_{i},\left.\boldsymbol{\lambda}\right|_{\mathbf{p}}, t_{n+1}, \mathbf{p}+\Delta \mathbf{p}_{i}\right) .
\end{aligned}
$$

To evaluate the terms $d \dot{\mathbf{q}} / d p_{i}$ and $d \ddot{\mathbf{q}} / d p_{i}$, we solve (8) for $\mathbf{a}_{n+1}$ and substitute that result into (9)-(10). Then, we differentiate the resulting two equations by $p_{i}$, use $d \mathbf{q}_{n+1} / d p_{i}=\mathbf{0}$, and solve for

$$
\begin{aligned}
\frac{d \dot{\mathbf{q}}_{n+1}}{d p_{i}} & =\frac{d \dot{\mathbf{q}}_{n}}{d p_{i}}+h(1-\gamma) \frac{d \mathbf{a}_{n}}{d p_{i}}+h \gamma \frac{d \mathbf{a}_{n+1}}{d p_{i}}, \\
\frac{d \ddot{\mathbf{q}}_{n+1}}{d p_{i}} & =\frac{\left(-1+\alpha_{m}\right) \frac{d \mathbf{a}_{n+1}}{d p_{i}}-\alpha_{m} \frac{d \mathbf{a}_{n}}{d p_{i}}+\alpha_{f} \frac{d \ddot{\mathbf{q}}_{n}}{d p_{i}}}{-1+\alpha_{f}}
\end{aligned}
$$

where the "predictor" derived from (8) is defined as

$$
\frac{d \mathbf{a}_{n+1}}{d p_{i}}=\frac{-1}{\beta h^{2}}\left(\left(\frac{1}{2}-\beta\right) h^{2} \frac{d \mathbf{a}_{n}}{d p_{i}}+h \frac{d \dot{\mathbf{q}}_{n}}{d p_{i}}+\frac{d \mathbf{q}_{n}}{d p_{i}}\right) .
$$

The derivative $\partial \boldsymbol{\Phi} / \partial p_{i}$ is similarly approximated as

$\frac{\partial \boldsymbol{\Phi}}{\partial p_{i}} \approx \frac{1}{\Delta p_{i}} \boldsymbol{\Phi}_{\text {pert }}$

where $\boldsymbol{\Phi}_{\text {pert }}=\boldsymbol{\Phi}\left(\left.\mathbf{q}\right|_{\mathbf{p}}, \mathbf{p}+\Delta \mathbf{p}_{i}\right)$.

Summarizing, the linear pseudo problem (28) reads

$-\mathbf{S}_{t}\left[\begin{array}{l}d \mathbf{q} / d p_{i} \\ d \boldsymbol{\lambda} / d p_{i}\end{array}\right]=\left[\begin{array}{c}\frac{1}{\Delta p_{i}} \mathbf{r}_{\text {pert }} \\ \frac{k}{\Delta p_{i}} \boldsymbol{\Phi}_{\text {pert }}\end{array}\right]$,

where $\mathbf{S}_{t}$ is the tangent iteration matrix defined in (20). Thusly, while many Newton iterations are required to evaluate the primal response $\mathbf{q}_{n}$, only $n_{v}$ back-solves are required to obtain its derivatives $d \mathbf{q}_{n+1} / d p_{i}$.

As in the primal analysis, the velocity and acceleration response derivative corrections must be added afterwards to satisfy the Newmark integration formulae, i.e.

$$
\begin{aligned}
\frac{d \dot{\mathbf{q}}_{n+1}}{d p_{i}} & =\frac{d \dot{\mathbf{q}}_{n+1}}{d p_{i}}+\frac{\gamma}{\beta h} \frac{d \mathbf{q}_{n+1}}{d p_{i}}, \\
\frac{d \ddot{\mathbf{q}}_{n+1}}{d p_{i}} & =\frac{d \ddot{\mathbf{q}}_{n+1}}{d p_{i}}+\frac{1}{\beta h^{2}} \frac{1-\alpha_{m}}{1-\alpha_{f}} \frac{d \mathbf{q}_{n+1}}{d p_{i}} .
\end{aligned}
$$


Finally, the "predictor" is updated from (8) as

$\frac{d \mathbf{a}_{n+1}}{d p_{i}}=\frac{d \mathbf{a}_{n+1}}{d p_{i}}+\frac{1}{\beta h^{2}} \frac{d \mathbf{q}_{n+1}}{d p_{i}}$.

We note that the initial condition derivatives $d \mathbf{q}_{0} / d p_{i}$, $d \dot{\mathbf{q}}_{0} / d p_{i}, d \ddot{\mathbf{q}}_{0} / d p_{i}$ and $d \mathbf{a}_{0} / d p_{i}$ are known.

From an implementation point of view, MBS simulation codes already have a function to compute the residual. Therefore, the perturbed residual in (32) can easily be evaluated by calling this function with the perturbed arguments.

The tangent iteration matrix $\mathbf{S}_{t}$ may or may not need to be recomputed depending on the convergence tolerance in the Newton-Raphson iterations. If the convergence criteria is quite small, one can reasonably use the tangent iteration matrix of the converged Newton iteration (i.e. without recomputing it with the updated values $\mathbf{q}_{n+1}$, etc.) to evaluate the sensitivity. Moreover, the tangent iteration matrix $\mathbf{S}_{t}$ has already been factorized during the primal analysis so the sensitivity analysis only requires $n_{v}$ back substitutions.

The procedure to obtain the sensitivities is described in Algorithm 2 which is called after the primal analysis at time step $t_{n+1}$ is computed. For the sake of notational compactness, we denote differentiation by $p_{i}$ via a comma, e.g. $d \mathbf{q}_{n+1} / d p_{i}=\mathbf{q}_{n+1, i}$. As seen in the algorithm, at each time step the cost to evaluate the sensitivities requires $n_{v}$ function calls and back-solves to compute the perturbed residuals $\mathbf{r}_{\text {pert }}$ and $\boldsymbol{\Phi}_{\text {pert }}$ and derivatives $\mathbf{q}_{n+1, i}$ and $\boldsymbol{\lambda}_{n+1, i}$.

As an aside, we note that a central finite difference can also be used to approximate the derivative of (30) to obtain increased accuracy. The authors recommend its use in the present case as the residual is highly nonlinear. We also note that the convergence criterion in the primal analysis must be very small to obtain accurate sensitivities since the basic assumption is $\left.\mathbf{r}\right|_{\left(t_{n+1}, \mathbf{p}\right)}=\mathbf{0}$.

\section{Slider-crank mechanism optimization}

\subsection{Problem description}

The first example concerns to the optimization of a connecting rod in a slider-crank mechanism of a fourstroke internal combustion diesel engine. The connecting rod is made of steel with a Young's Modulus of $\mathrm{E}=210 \mathrm{GPa}$, Poisson's ratio of $\nu=0.3$ and mass density of $\rho=7800 \mathrm{~kg} / \mathrm{m}^{3}$. It is driven by the crankshaft at $4000 \mathrm{rpm}$. At this high rotation speed, the dynamic loading due to inertia forces represents about $9 \%$ of the loading at the top dead center i.e. detonation, configuration.

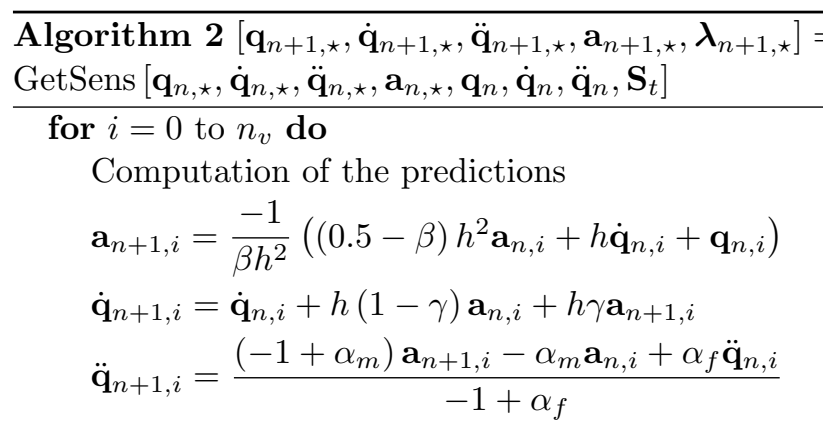

Computation of the perturbed residuals $\mathbf{r}_{\text {pert }}$ and $\boldsymbol{\Phi}_{\text {pert }}$

System resolution

$\left[\begin{array}{c}\mathbf{q}_{n+1, i} \\ \boldsymbol{\lambda}_{n+1, i}\end{array}\right]=-\mathbf{S}_{t}^{-1}\left[\begin{array}{c}\frac{1}{\Delta p_{i}} \mathbf{r}_{\text {pert }} \\ \frac{k}{\Delta p_{i}} \boldsymbol{\Phi}_{\text {pert }}\end{array}\right]$

Update

$$
\begin{aligned}
\dot{\mathbf{q}}_{n+1, i} & =\dot{\mathbf{q}}_{n+1, i}+\frac{\gamma}{\beta h} \mathbf{q}_{n+1, i} \\
\ddot{\mathbf{q}}_{n+1, i} & =\ddot{\mathbf{q}}_{n+1, i}+\frac{1}{\beta h^{2}} \frac{1-\alpha_{m}}{1-\alpha_{f}} \mathbf{q}_{n+1, i} \\
\mathbf{a}_{n+1, i} & =\mathbf{a}_{n+1, i}+\frac{1}{\beta h^{2}} \mathbf{q}_{n+1, i}
\end{aligned}
$$

\section{end for}

Remark: The index $\star$ indicates that the term has a $2 \mathrm{D}$ matrix structure where column $i$ corresponds to the derivative of the variable with respect to the design variable $p_{i}$.

The numerical simulation is conducted by imposing the angular rotation of the crankshaft. First, the crankshaft is accelerated from 0 to $4000 \mathrm{rpm}$ in $0.01 \mathrm{~s}$. After a period of $2.5 \times 10^{-3} \mathrm{~s}$, initial transients dissipate and steady response is obtained. The rotation speed of $4000 \mathrm{rpm}$ is maintained when the gas pressure is applied over the piston face over the $720^{\circ}$ engine cycle using experimentally obtained pressure data. The generalized- $\alpha$ with a time step of $2.5 \times 10^{-4} \mathrm{~s}$ and a spectral radius $\rho_{\infty}=0.8$ is used for the time integration.

A simplifying 2D model of the connecting rod is considered in this study (Figs. 2 and 3). It is modeled by plane stress elements using a transfinite mesh $(10 \mathrm{~mm}$ thick). The crankshaft is considered rigid while the piston is represented by a point mass of $0.456 \mathrm{~kg}$. The components are linked with ideal kinematic cylindrical and sliding joints. Rigid connections are employed to link the small and big ends of the connecting rod to their respective revolute joints.

To obtain the highest compression ratio and hence engine power, the distance between the piston and the valves must be as small as possible at top dead cen- 


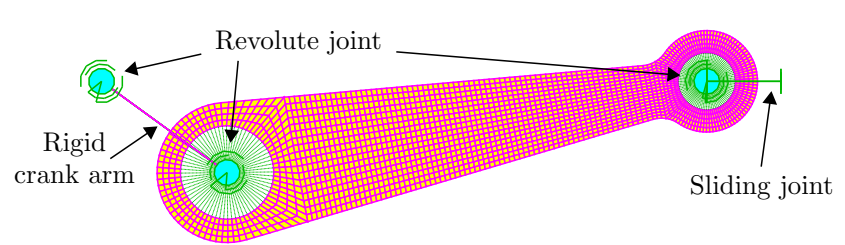

Fig. 2 Finite element model of the slider-crank mechanism.

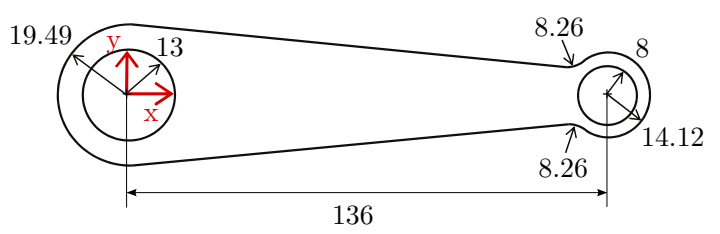

Fig. 3 Geometry and dimensions of the connecting rod in $\mathrm{mm}$.

ter. Because of this, the elongation of the connecting rod must be accurately controlled to avoid piston-valve interference. At the same time it is desirable to reduce engine weight, so the goal of the optimization is to minimize mass $m$ subject to elongation constraints.

In this connecting rod optimization, internal holes are defined with an implicit level let description and optimized whereas the external contour is defined via a traditional explicit formulation and held fixed. However, it is noted that the entire geometry can be described using level set functions.

\subsection{An elliptical hole}

The first optimization problem introduces an elliptical hole at the nearby center location $\mathbf{c}=(67.8,0) \mathrm{mm}$ of the connecting rod (Fig. 4). The level set function is defined as

$\phi(\mathbf{x}, p)=\sqrt{\frac{\left(x-c_{x}\right)^{2}}{a^{2}}+\frac{\left(y-c_{y}\right)^{2}}{b^{2}}}-p$,

where $\mathbf{x}=(x, y)$, the parameters $a=0.05$ and $b=0.3$ are constant and $p$ represents the sole design variable. It is constrained such that $p \in(\underline{p}, \bar{p})=(0.001,0.15)$.

The function $f_{n}(\mathbf{q}, p)$ quantifies the connecting rod deformation at time step $t_{n}$. It is equal to the difference of the Euclidean distances between the rod small end center and the rod large end center in the deformed and undeformed configurations. To avoid piston-valve interference, we enforce the constraint $f_{n}(\mathbf{q}, p)<\Delta l_{\max }$ where $\Delta l_{\max }=16 \mu \mathrm{m}$ is the elongation upper bound.

To optimize the connecting rod, we adopt a local formulation which considers the response at each time step of the MBS simulation. This local optimization problem formulation minimizes the connecting rod mass $m(p)$

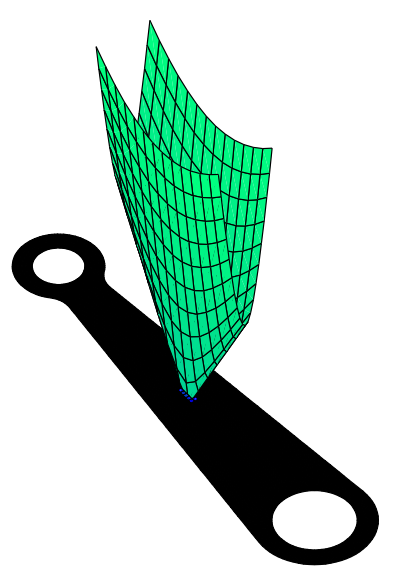

Fig. 4 Representation of the elliptical level set function.

while constraining $f_{n}(\mathbf{q}, p)$ at each time step $t_{n}$. Mathematically, it is stated as

$\underset{p \in(\underline{p}, \bar{p})}{\operatorname{minimize}} m(p)$

subject to $\quad f_{n}(\mathbf{q}, p) \leq \Delta l_{\max }, \quad$ for $n=1, \ldots, n_{\text {end }}$

where $n_{\text {end }}$ is the terminal analysis time step.

Initially, the design variable $p$ is set to its lower bound making the hole tiny and the initial design stiff. Thusly, the initial design satisfies all the constraint functions. The connecting rod has a corresponding mass of $295.7 \mathrm{~g}$ and a maximal elongation of $13.51 \mu \mathrm{m}$.

The optimization process is deemed converged when the objective function varies less than $0.01 \%$ between iterations. This condition is attained after 7 iterations resulting in an $8.2 \%$ mass reduction. Figures 5 and 6 show the convergence history and optimal design. Numerical results are gathered in Table 1 .

It is observed that the optimization process converges monotonically towards the elongation upper bound. Figure 5 illustrates the history of the elongation constraint maximal value.

Table 1 Optimization results - An elliptical hole.

\begin{tabular}{ccc}
\hline & Initial value & Optimal value (bounds) \\
\hline Mass, g & 295.664 & 271.337 \\
Elongation, $\mu \mathrm{m}$ & 13.508 & 15.996 \\
Design variable $p$ & 0.001 & $0.0819(0.001-0.15)$ \\
\hline
\end{tabular}

Although the optimized geometry does not appear clearly in Figure 6(a), keep in mind that the boundary of the hole is defined by CAD entities, and thusly it is straightforward to manufacture. Indeed, there is no 


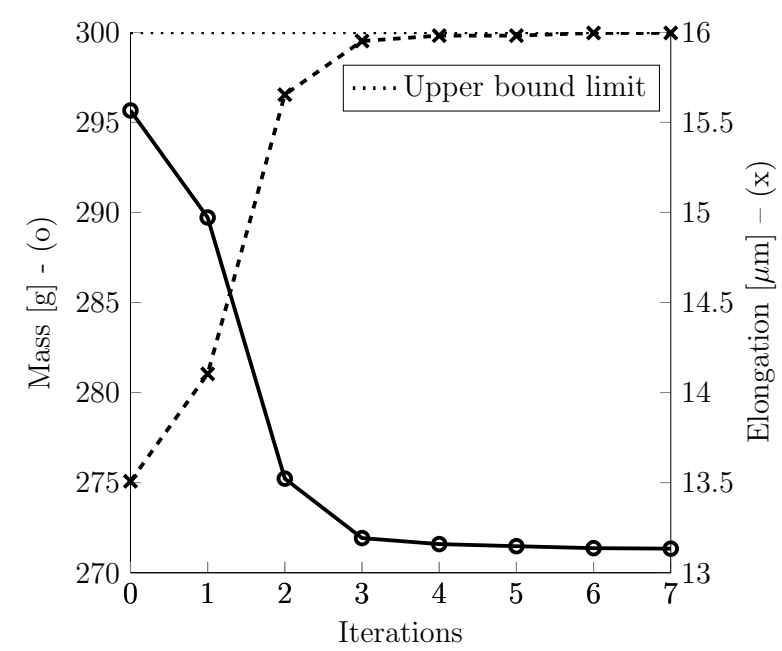

Fig. 5 Evolution of the mass and the maximal connecting rod elongation with an elliptical hole at its center.

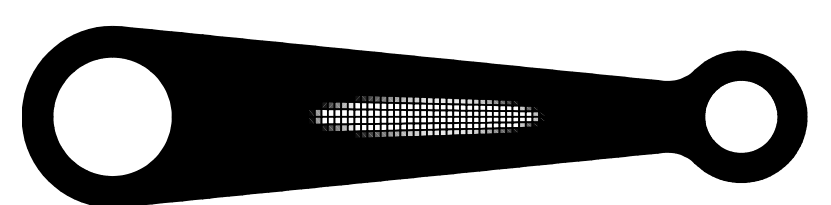

(a) Mesh resulting from the optimization process.

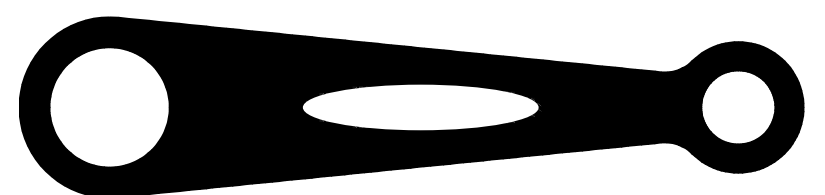

(b) CAD model.

Fig. 6 Optimal shape of the connecting rod with an elliptical hole at its center.

need to interpret the resulting design since the geometry is defined via the elliptical level set feature (Fig. 6(b)).

\subsection{Topology modification}

The second example introduces three elliptical level set features which are combined using the boolean $A N D$ operation to improve the optimal design of the previous example. This parameterization offers the possibility of merging three elliptical holes.

Referring to (41), the design variables $p_{i}$ are the level set parameters denoted in Table 2. To maintain design symmetry, level sets 2 and 3 are symmetrically centered with respect to the $x$-axis.

The optimization problem is identical to (42) with the exception that we now have five design variables. Initially, the features are located nearby the optimal hole obtained in the previous example. However, the
Table 2 Parameter values of the level set functions.

\begin{tabular}{cccccc}
\hline & $c_{x}$ & $c_{y}$ & $a$ & $b$ & $d$ \\
\hline Level set 1 & $p_{3}$ & 0 & 0.06 & 0.3 & $p_{1}$ \\
Level set 2 & $p_{5}$ & $-p_{4}$ & 0.045 & 0.28 & $p_{2}$ \\
Level set 3 & $p_{5}$ & $p_{4}$ & 0.045 & 0.28 & $p_{2}$ \\
\hline
\end{tabular}

holes size are small initially making a stiff connecting rod which satisfies all the constraints.

Convergence using the criterion from the previous example is attained after 18 iterations (Fig. 7). Due to the highly nonlinear behavior of the optimization problem, constraint violations are observed during the initial iterations. Table 3 gathers the numerical results where it is seen, as expected, that the mass reduction is larger than the previous example since the design has more freedom.

Figure 8 illustrates the volume fraction history where it is seen that three holes merge into one single large hole. This example illustrates the level set method's ability to merge, remove and separate entities. It also illustrates the method's ability to evolve topology which we attribute to the implicit geometry representation over a fixed mesh and to the boolean coupling of the different geometric features. Such design modifications cannot be achieved by classical shape optimization using CAD descriptions wherein the component topology is fixed during the optimization.

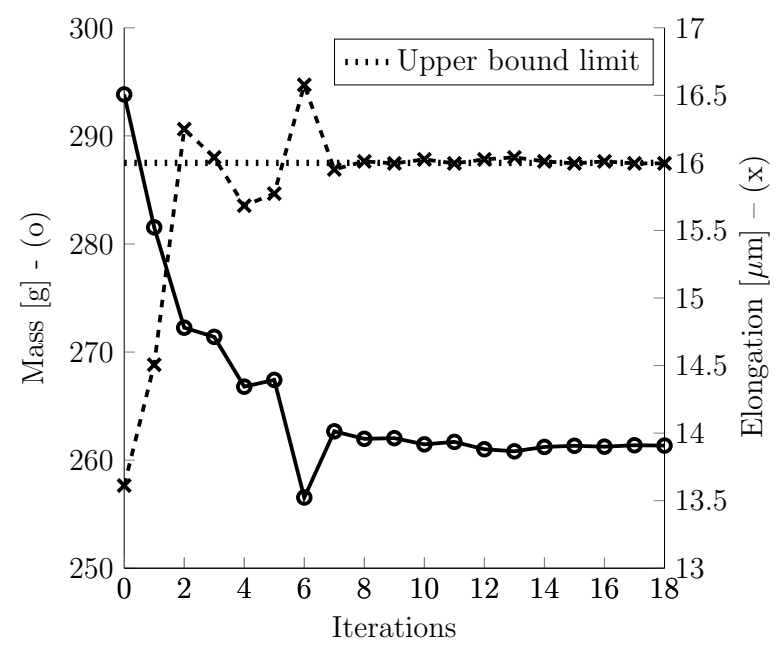

Fig. 7 Evolution of the mass and the connecting rod elongation considering 3 level set functions. 
Table 3 Optimization results.

\begin{tabular}{ccc}
\hline & Initial Value & Optimal value (bounds) \\
\hline Mass, g & 293.832 & 261.348 \\
Elongation, $\mu \mathrm{m}$ & 13.612 & 15.996 \\
$p_{1}$ & 0.01 & $0.0573(0-0.08)$ \\
$p_{2}$ & 0.015 & $0.0761(0-0.095)$ \\
$p_{3}$ & 0.0875 & $0.06906(0.0668-0.0968)$ \\
$p_{4}$ & 0.003 & $0.000973(0-0.004)$ \\
$p_{5}$ & 0.0493 & $0.0430(0.0418-0.0718)$ \\
\hline
\end{tabular}

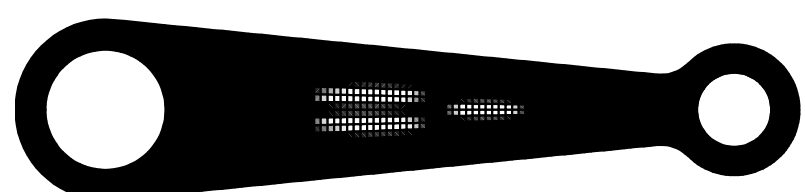

(a) Iteration 2 .

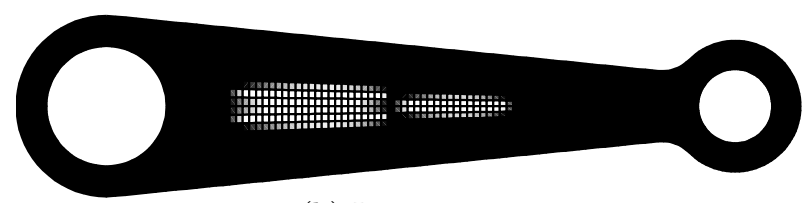

(b) Iteration 5 .

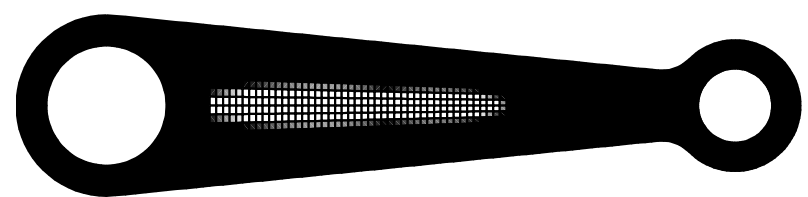

(c) Iteration 18 - Optimal design.

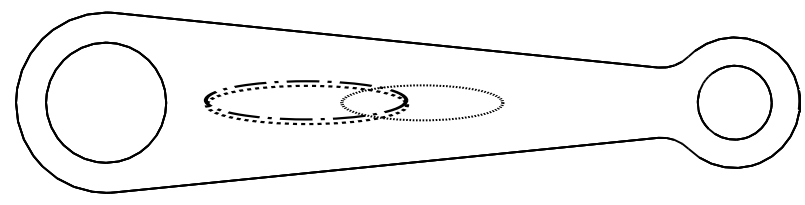

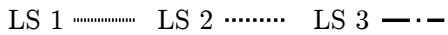

(d) Iteration 18 - Level set position.

Fig. 8 Topology evolution of the connecting rod.

\section{Robot arm optimization}

\subsection{Problem description}

The race to reduce production costs is clearly under way. In robotics this reduction is obtained by minimizing their moving mass which decreases energy requirements and/or increases their working speed. Unfortunately, reducing mass increases flexibility leading to larger vibrations which adversely affect the precision of the robot.

With this background in mind, we minimize the mass of a planar robot while simultaneously controlling the deviation of the tip trajectory from a prescribed path. Figure 9 illustrates the kinematic model of the robot, inspired from Brüls et al (2011), which moves in the horizontal plane so that gravity can be ignored. An effector with $5 \mathrm{~kg}$ mass is fixed at the tip of the manipu- lator. Each robot arm has a length of $1 \mathrm{~m}$. The first arm is approximated as a rigid body while the second one is flexible and modeled using a rectangular design domain and plane stress assumption. It is made of aluminum with a Young's modulus of E $=72 \mathrm{GPa}$, Poisson's ratio of $\nu=0.3$ and mass density of $\rho=2700 \mathrm{~kg} / \mathrm{m}^{3}$. Figure 10 gives more details about the finite element modeling where it is seen that rigid rods link the rectangular link to the joint and tip.

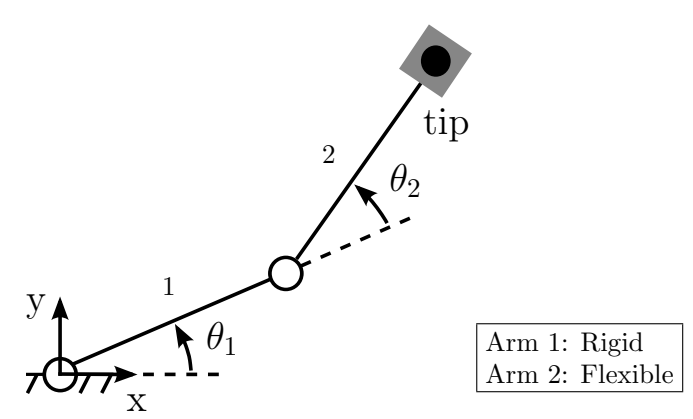

Fig. 9 Kinematic model of the 2-dof robot.

An out-of-plane thickness $e=2.5 \mathrm{~mm}$ is assigned to the plane stress body. We note that the $0.05 \mathrm{~m}$ inplane width of the rectangular robotic arm is slender compared to its $0.6 \mathrm{~m}$ length. As a consequence, the robot arm undergoes significant vibrations.

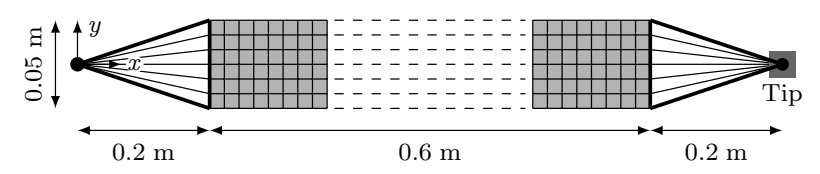

Fig. 10 Modeling of the flexible arm. (This figure is not to scale.)

Each revolute joint is driven by an ideal motor which imposes a smooth joint trajectory $\theta_{1}(t)$ and $\theta_{2}(t)$ as illustrated in Figure 11. The point-to-point trajectory is composed of an acceleration phase, a constant speed phase and a deceleration phase. The initial and terminal joint angles are $\theta_{1 i}=\theta_{2 i}=\pi / 3 \mathrm{rad}, \theta_{1 f}=$ $\theta_{2 f}=\pi / 2 \mathrm{rad}$, the joints acceleration (deceleration) is $1 \mathrm{rad} / \mathrm{s}^{2}$ and the constant speed is $0.25 \mathrm{rad} / \mathrm{s}$. The simulation is performed using the generalized- $\alpha$ scheme with a time step $h=0.005 \mathrm{~s}$ and a spectral radius $\rho_{\infty}=0.8$. 


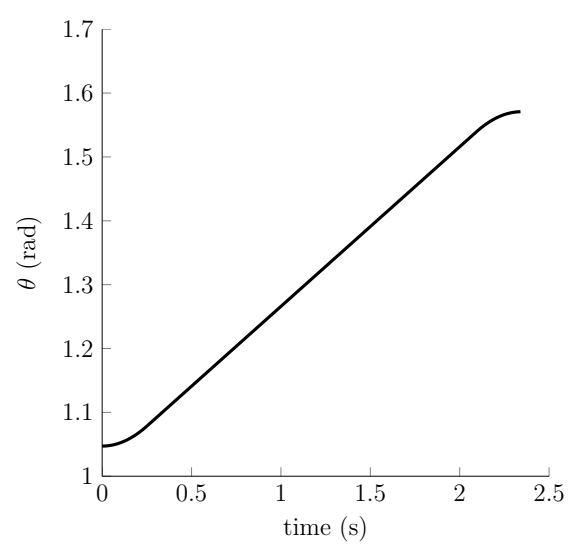

Fig. 11 Imposed joint trajectory for $\theta_{1}$ and $\theta_{2}$.

\subsection{Optimization problem and numerical results}

The design variables $p_{i}$ in this optimization are the parameters that describe 5 super-ellipses defined as follows

$\phi(\mathbf{x}, \mathbf{p})=\left|\frac{\left(x-c_{x}\right)}{a}\right|^{\xi}+\left|\frac{\left(y-c_{y}\right)}{b}\right|^{\eta}-1$.

where e.g. $p_{1}=c_{x}, p_{2}=a$ and $p_{3}=b$ for the first level set function. Similar variables are assigned to the remaining 4 level set functions for a total of $n_{v}=15$ design variables. The lower $\mathbf{p}$ and upper $\overline{\mathbf{p}}$ bounds of the design variables $a$ and $b$ are $\overline{0} .0001$ and 0.04 respectively while bounds of 0.25 and 0.75 are placed on $c_{x}$. Without loss of generality, $\xi=\eta=5$ are constant as is the variable $c_{y}=0$ which centers the super-ellipses over the width (cf. the coordinate system defined in Figure 10).

The constraint is similar to (42) except here the function $f_{n}(\mathbf{q}, \mathbf{p})$ represents the tip deviation from the desired trajectory $\mathbf{q}_{n}^{\text {pres }}$ and that of the design, i.e.

$f_{n}(\mathbf{q}, \mathbf{p})=\left\|\mathbf{q}_{n}^{\text {pres }}-\mathbf{q}_{n}^{\text {tip }}(\mathbf{p})\right\|$

where $\mathbf{q}_{n}^{\text {tip }}$ denotes the tip position. Moreover, here we use a global formulation since the considered application does not require high precision at each time step, but rather must satisfy an "average" precision error. As such, the optimization problem is stated as

$$
\begin{array}{ll}
\underset{\mathbf{p} \in(\underline{\mathbf{p}}, \overline{\mathbf{p}})}{\operatorname{minimize}} & m(\mathbf{p}) \\
\text { subject to } & \frac{1}{n_{c v f}-n_{c v i}} \sum_{n=n_{c v i}}^{n_{c v f}} f_{n}(\mathbf{q}, \mathbf{p}) \leq 0.001,
\end{array}
$$

where $n_{c v i}$ and $n_{c v f}$ are respectively the initial and final time step of the constant speed phase.

The optimization is deemed converged when the relative change of the objective function value and relative constraint violation are less than $0.05 \%$ and $0.25 \%$ respectively.

Figure 12 illustrates large oscillations in the objective and constraint function values during the initial design iterations. We attribute this behavior to large design variable changes and the highly nonlinear dynamic response. As the design changes lessen, the nonlinear effects are subdued and smooth convergence is observed. Move limit strategy may be implemented to prevent the oscillations.

Table 4 presents the optimization results while Table 5 gives the initial and optimal values of the design variables. The features reach their maximal dimensions to create the largest holes and move towards the arm extremity where some features merge. Larger holes are thus created but they are separated by thin solid parts (ties) to retain the flexural stiffness while minimizing the mass. Figure 13 shows the optimal design of the flexible arm. In comparison with the results presented in Brüls et al (2011) which treats the arm as a truss structure, it is observed that the topology is similar insofar as the region near the joint is quite stiff while the region near the tip contains several holes.

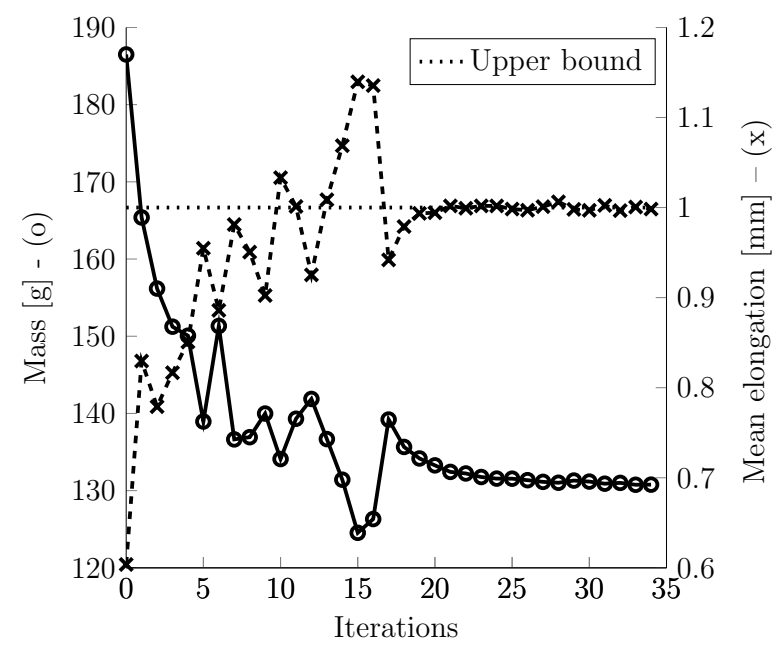

Fig. 12 Convergence history of the optimization process.

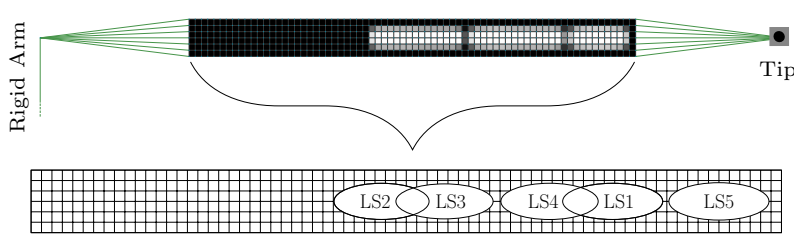

Fig. 13 Optimal design of the flexible arm. 
Table 4 Optimization results of the 2-dof robot.

\begin{tabular}{ccc}
\hline & Initial Value & Optimal value \\
\hline Mass (flexible arm), g & 186.498 & 130.778 \\
Average deviation, mm & 0.6037 & 0.9983 \\
\hline
\end{tabular}

Table 5 Parameter values of the 2-dof robot.

\begin{tabular}{ccccccc}
\hline & \multicolumn{4}{c}{ Initial values } & \multicolumn{3}{c}{ Optimal values } \\
& $a$ & $b$ & $c_{x}$ & $a$ & $b$ & $c_{x}$ \\
\hline LS 1 & 0.01 & 0.01 & 260 & 39.99 & 14.47 & 665.03 \\
LS 2 & 0.01 & 0.01 & 380 & 38.04 & 14.46 & 480.18 \\
LS 3 & 0.01 & 0.01 & 500 & 38.85 & 13.99 & 530.56 \\
LS 4 & 0.01 & 0.01 & 620 & 38.89 & 14.49 & 614.54 \\
LS 5 & 0.01 & 0.01 & 740 & 39.99 & 14.94 & 749.99 \\
\hline
\end{tabular}

\section{Conclusions and perspectives}

The present paper is devoted to a level set geometric description for the optimization of structural components in flexible multibody systems. This approach enables a generalized shape optimization that encompasses the advantages of shape and topology optimizations.

The examples illustrate the method's ability to accommodate new geometric features and topology. Moreover, the CAD model that is used to generate the initial design is not required. This is extremely useful for industrial applications involving confidentiality wherein only the finite element mesh is shared, not the proprietary CAD model. Indeed, only the level set description of the geometry modifications is required to perform the optimization. The optimal design can later be combined with the proprietary CAD model. Although only basic geometric features, e.g. ellipses or super-ellipses, have been employed in the present study, others can be incorporated without any difficulties. That said, these results have been validated against other papers.

The adopted level set geometrical description is based on common entities (circle, ellipse, NURBS...), which are commonplace in manufacturing. This contrasts designs obtained via topology optimization which require various means to recreate $\mathrm{CAD}$ models.

Another major contribution of this paper concerns the sensitivity analysis method. The proposed method leverages the existing MBS analysis by re-using its already computed and factorized tangent matrices from the primal analysis and its ability to compute the system residual. Ultimately, the sensitivity analysis cost is limited to a few function calls and back-solves which can be readily incorporated into the existing MBS analysis code.
In the future, it would be interesting to extend this approach to $3 \mathrm{D}$ problems and to consider stress constraints.

Acknowledgements The first author wishes to acknowledge the LIGHTCAR Project sponsored by the pole of competitiveness "Mecatech" and the Walloon Region of Belgium for their supports (Contract RW-6500).

\section{References}

Arnold M, Brüls O (2007) Convergence of the generalized$\alpha$ scheme for constrained mechanical systems. Multibody Systems Dynamics 18(2):185-202

Bendsøe M, Kikuchi N (1988) Generating optimal topologies in optimal design using a homogenization method. Computational methods in applied mechanics and engineering $71: 197-224$

Bendsøe M, Sigmund O (2003) Topology optimization: Theory, Methods, and Applications. Springer Verlag, Berlin

Bestle D, Seybold J (1992) Sensitivity analysis of constrained multibody systems. Archive of Applied Mechanics 62:181-190

Brüls O, Eberhard P (2008) Sensitivity analysis for dynamic mechanical systems with finite rotations. International Journal for Numerical Methods in Engineering 74(13):1897-1927

Brüls O, Lemaire E, Duysinx P, Eberhard P (2011) Optimization of multibody systems and their structural components. In: Multibody Dynamics: Computational Methods and Applications, vol 23, Springer, pp 49-68

Bruns T, Tortorelli D (1995) Computer-aided optimal design of flexible mechanisms. In: Proceedings of the Twelfth Conference of the Irish Manufacturing Commitee, IMC12, Competitive Manufacturing, University College Cork, Ireland

Chung J, Hulbert G (1993) A time integration algorithm for structural dynamics with improved numerical dissipation: The generalized- $\alpha$ method. Journal of applied mechanics 60:371-375

Deaton J, Grandhi R (2014) A survey of structural and multidisciplinary continuum topology optimization: post 2000. Structural and Multidisciplinary Optimization 49(1):1-38

Duysinx P, Zhang W, Zhong H, Beckers P, Fleury C (1994) Structural shape optimization with error control. In: Proceedings of the 20th ASME Design Automation Conference, Mineapolis, USA

Géradin M, Cardona A (2001) Flexible Multibody Dynamics: A Finite Element Approach. John Wiley \& Sons, New York

Haftka R, Grandhi R (1986) Structural shape optimization - a survey. Computer methods in applied mechanics and engineering 57:91-106

Häussler P, Emmrich D, Müller O, Ilzhöfer B, Nowicki L, Albers A (2001) Automated topology optimization of flexible components in hybrid finite element multibody systems using ADAMS/Flex and MSC.Construct. In: Proceedings of the 16th European ADAMS Users' Conference

Häussler P, Minx J, Emmrich D (2004) Topology optimization of dynamically loaded parts in mechanical systems: Coupling of MBS, FEM and structural optimization. In: Proceedings of NAFEMS Seminar Analysis of Multi-Body Systems Using FEM and MBS, Wiesbaden, Germany 
Hong E, You B, Kim C, Park G (2010) Optimization of flexible components of multibody systems via equivalent static loads. Structural Multidisciplinary Optimization 40:549-562

Kang B, Park G, Arora J (2005) Optimization of flexible multibody dynamic systems using the equivalent static load method. AIAA Journal 43(4):846-852

Kim N, Chang Y (2005) Eulerian shape design sensitivity analysis and optimization with a fixed grid. Computer methods in applied mechanics and engineering 194:32913314

Norato J, Haber R, Tortorelli D, Bendsøe M (2004) A geometry projection method for shape optimization. International Journal for Numerical Methods in Engineering 60:2289-2312

Oral S, Kemal Ider S (1997) Optimum design of high-speed flexible robotic arms with dynamic behavior constraints. Computers \& Structures 65(2):255-259

Osher S, Sethian J (1988) Fronts propagating with curvaturedependent speed: Algorithms based on Hamilton-Jacobi formulations. Journal of Computational Physics 79:12-49

Schleupen A, Maute K, Ramm E (2000) Adaptive feprocedures in shape optimization. Structural and Multidisciplinary Optimization 19(4):282-302

Seifried R, Held A (2011) Integrated design approaches for controlled flexible multibody systems. In: Proceedings of the ASME 2011 International Design Engineering Technical Conferences \& Computers and Information in Engineering Conference (IDETC/CIE 2011), Washington DC, USA

Shapiro V (2007) Semi-analytic geometry with r-functions. Acta Numerica 16:239-303

Sherif K, Irschik H (2010) Efficient topology optimization of large dynamic finite element systems using fatigue. AIAA Journal 48(7):1339-1347

Sigmund O, Maute K (2013) Topology optimization approaches: A comparative review. Structural and Multidisciplinary Optimization 48(6):1031-1055

Svanberg K (1987) The method of moving asymptotes - a new method for structural optimization. International Journal for Numerical Methods in Engineering 24:359-373

Tobias C, Fehr J, Eberhard P (2010) Durability-based structural optimization with reduced elastic multibody systems. In: Proceedings of $2^{\text {nd }}$ International Conference on Engineering Optimization, Lisbon, Portugal

Tromme E, Brüls O, Emonds-Alt J, Bruyneel M, Virlez G, Duysinx P (2013) Discussion on the optimization problem formulation of flexible components in multibody systems. Structural Multidisciplinary Optimization 48:1189-1206

Van Dijk N, Maute K, Langelaar M, Van Keulen F (2013) Level-set methods for structural topology optimization: a review. Structural Multidisciplinary Optimization 48:437-472

Van Keulen F, Haftka R, Kim N (2005) Review of options for structural design sensitivity analysis. Part 1: Linear systems. Computer Methods in Applied Mechanics and Engineering 194:3213-3243

Van Miegroet L, Duysinx P (2007) Stress concentration minimization of $2 \mathrm{D}$ filets using $\mathrm{x}$-fem and level set description. Structural Multidisciplinary Optimization 33:425-438 